\title{
TAGUNG
}

\section{Quo vadis, europäisches Gesellschaftsrecht?}

\author{
Sabina Krispenz und Ann Seibert*
}

Einleitend wiesen Peter-Christian MüllerGraff und Christoph Teichmann auf zwei vieldiskutierte Entwicklungen im unmittelbaren Vorfeld der Tagung ,Europäisches Gesellschaftsrecht auf neuen Wegen“" hin, welche die hohe Aktualität des europäischen Gesellschaftsrechts vor Augen führen. Der Vorschlag der Europäischen Kommission für eine Verordnung zur Europäischen Privatgesellschaft und die kürzlich vom Bundestag beschlossenen Änderungen des GmbH-Gesetzes $^{1}$ machten deutlich, dass sich das Gesellschaftsrecht sowohl auf nationaler als auch auf europäischer Ebene im Wandel befinde. Dabei seien nicht nur Detailfragen, wie zum Beispiel die Ausgestaltung europäischer Gesellschaftsformen, im Einzelnen umstritten. Auch die Grundlagen des europäischen Gesellschaftsrechts, darunter vor allem die Frage nach Notwendigkeit und Inhalt eines einheitlichen Gesellschaftskollisionsrechts, bedürften noch weitgehend der Klärung.

Die rechtlichen Grundlagen des europäischen Gesellschaftsrechts

Peter-Christian Müller-Graff erläuterte die Grundlagen des europäischen Gesellschaftsrechts vor dem Hintergrund des Wirtschaftsordnungsrechts der Europäischen Gemeinschaft. Wesentlich sei insoweit die Verpflichtung der Gemeinschaft $\mathrm{zu}$ einer freien und offenen Marktwirtschaft, die in dem ,fein gewirkten System der binnen-

\section{Europäisches Gesellschaftsrecht auf neuen Wegen}

Tagung des Arbeitskreises Europäische Integration e.V. in Verbindung mit dem Institut für deutsches und europäisches Gesellschaftsund Wirtschaftsrecht der Universität Heidelberg

$$
\begin{gathered}
\text { mit Unterstützung der Europäischen } \\
\text { Kommission }
\end{gathered}
$$

Heidelberg, 11./12. Juli 2008

\section{Wissenschaftliche Leitung:}

Prof. Dr. Dr. h.c. Peter-Christian MÜLLER-

GRAFF, Direktor des Instituts für deutsches und europäisches Gesellschafts- und Wirtschaftsrecht, Universität Heidelberg

Prof. Dr. Christoph TEICHMANN, Institut für Gesellschafts-, Steuer- und Arbeitsrecht, Universität Würzburg

Gesellschaftsrecht als Teil des sich entwickelnden Europäischen Binnenmarktrechts und Wirtschaftsordnungsrechts

Prof. Dr. Dr. h.c. Peter-Christian MÜLLER-

GRAFF, Universität Heidelberg

Wettbewerb der mitgliedstaatlichen Gesellschaftsgesetzgebungen als neues Element der Binnenmarktintegration

Prof. Dr. Christoph TEICHMANN, Universität Würzburg

Binnenmarktintegration durch uniformes Gesellschaftskollisionsrecht?

Prof. Dr. Eva-Maria KIENINGER, Universität Würzburg

* Sabina Krispenz, Wissenschaftliche Mitarbeiterin, Institut für deutsches und europäisches Gesellschafts- und Wirtschaftsrecht, Universität Heidelberg.

Ann Seibert, Wissenschaftliche Mitarbeiterin, Institut für deutsches und europäisches Gesellschafts- und Wirtschaftsrecht, Universität Heidelberg.

1 Gesetz zur Modernisierung des GmbH-Rechts und zur Bekämpfung von Missbräuchen (MoMiG). Vgl. Beschlussempfehlung des Rechtsausschusses (6. Ausschuss), in: Bundestagsdrucksache 16/9737 vom 24.06.2008. 
marktrechtlichen Grundfreiheiten" und dem europäischen Wettbewerbsrecht ihre Konkretisierung fände. Müller-Graff widmete sich zunächst den Auswirkungen der Einführung des Binnenmarktkonzepts durch die Einheitliche Europäische Akte und der daran anknüpfenden Rechtsprechung des Europäischen Gerichtshofes auf die Mobilität und die Ausgestaltung der Gesellschaftsformen. Die Zurückdrängung der Sitzlehre habe den Weg zu mehr Mobilität der Gesellschaften geöffnet. Einwände, die diese Entwicklung als vertragswidrig erscheinen lassen, überzeugen dabei nach Ansicht Müller-Graffs nicht. Auch hinsichtlich der Rechtssache Cartesio ${ }^{2}$ äußerte sich Müller-Graff optimistisch dahingehend, dass der Europäische Gerichtshof seine Linie zur Liberalisierung fortsetzen und die Wegzugsbeschränkungen als von Artikel 43 EGV umfasst ansehen werde. Auch die Ausgestaltung der Gesellschaftsformen werde durch die Grundfreiheiten und das Wettbewerbsrecht beeinflusst. Folgewirkungen ergäben sich daraus für den Mitgliedschaftserwerb, die Organzusammensetzung, die Kapitalverfassung und die Wettbewerbsverbote. Schließlich verdeutlichte Müller-Graff auch die Auswirkungen der Grundfreiheiten und des Wettbewerbsrechts auf die Ausgestaltung des europäischen Gesellschaftsrechts. So stehe das Wettbewerbsrecht für eine Förderung der Handlungsalternativen durch eine Vielzahl von Gesellschaftsformen, während die Grundfreiheiten die Verpflichtung zur Mobilität auch auf Ebene der europäischen Gesellschaftsformen beinhalten. Das grenzüberschreitende Zusammenwirken müsse erleichtert werden, was bei der angedachten Europäischen Privatgesellschaft durch den Verzicht auf ein transnationales Gründungselement verwirklicht werde. Doch dabei stellt sich nach MüllerGraff die Frage nach der Kompetenz der Gemeinschaft, die durch eine solche Ausgestaltung der Gesellschaftsform in den Wettbewerb mit den nationalen Gesetzgebern trete. Ergänzt würden die Vorgaben des Wirt-
Schutz der Gesellschaftsgläubiger im Binnenmarkt durch gesetzliches Mindestkapital und alternative Mittel

Prof. Dr. Hanno MERKT, Universität Freiburg

Steigerung der grenzüberschreitenden Unternehmensmobilität durch Richtlinien zur grenzüberschreitenden Verschmelzung und Sitzverlegung

Rechtsanwalt Dr. Rainer KRAUSE, Hengeler

Mueller, Düsseldorf

Die Europäische Aktiengesellschaft im

Freilandversuch der Praxis

Rechtsanwalt Dr. Jochem REICHERT, Schilling,

Zutt \& Anschütz, Mannheim

Mitbestimmung als Verhandlungsgegenstand grenzüberschreitender Strategien

Prof. Dr. Martin HENSSLER, Universität zu Köln

Aktionärsrechte als neuer Gegenstand des Europäischen Gemeinschaftsrechts

Prof. Dr. Thomas BACHNER, Wirtschaftsuniversität Wien

Der Projektstand der Europäischen Privatgesellschaft

Dr. Paulina DEJMEK, Juristischer Dienst, Europäische Kommission, Brüssel

Neue gemeinschaftsrechtliche Projekte zum europäischen privaten Verbandsrecht und Stiftungsrecht

Prof. Dr. Søren Friis HANSEN, Syddansk Universitet, Odense

schaftsordnungsrechts für die Ausgestaltung des Gesellschaftsrechts durch das Erforderlichkeitsprinzip und die Kompetenzverteilung zwischen den Mitgliedstaaten und der Gemeinschaft. So stehe der Europäischen Gemeinschaft auf dem Gebiet des Gesellschaftsrechts keine Bereichskompetenz zu. Vielmehr müssten die einzelnen Kompetenzen in Artikel 44 Absatz 2, 46 Absatz 2, 94, 95 und 308 EGV unter Beachtung der Erforderlichkeit und des Subsidiaritätsprinzips ge-

2 Siehe: Schlussanträge des Generalanwalts Poiares Maduro: Rs. C-210/06 (Cartesio Oktató és Szolgáltató bt), Vorabentscheidungsersuchen des Szegedi Ítélõtábla, 22.05.2008. 
funden werden. Abschließend stellte MüllerGraff fest, dass dem nationalen und europäischen Gesetzgeber auf dem Gebiet des Gesellschaftsrechts ein großer Gestaltungsspielraum offen steht. Das Wirtschaftsordnungsrecht der Gemeinschaft übe dabei eine Leitplankenfunktion aus und lasse eine Vielzahl von Gesellschaftsformen zu. Diese würden sich in Zukunft in einem offenen Kräftespiel gegeneinander behaupten müssen.

Christoph Teichmann erläuterte die Bedeutung des Wettbewerbsprinzips als Maßstab für den nationalen Gesetzgeber bei der Ausgestaltung der Gesellschaftsformen. Dabei widmete er sich der Frage, ob das Gemeinschaftsrecht den Wettbewerb der mitgliedstaatlichen Gesellschaftsgesetzgebungen fördert oder gar fordert. Ausgangspunkt bildete dabei die Darstellung der Entwicklung der Rechtslage im US-Bundesstaat Delaware. Teichmann führte zunächst aus, wie sich der Wettbewerb der Gesellschaftsrechtsgesetzgebungen in den USA entwickelte und warum Delaware sich als besonders attraktiv für Gesellschaften erweist. So sei dabei das besonders gut funktionierende Rechtspflegesystem ausschlaggebend. Daneben bilde aber auch die Existenz einer zentralen Instanz in Form des Bundesgesetzgebers, als Gegengewicht zu den einzelstaatlichen Versäumnissen, einen entscheidenden Faktor. Allerdings äußerte Teichmann Zweifel daran, ob diese Gesetzmäßigkeiten ohne Weiteres auf die Entwicklungen in Europa übertragbar sind. Denn vor allem der fehlende Zusammenhang zwischen der Reformgesetzgebung in den einzelnen Mitgliedstaaten und dem Gründungsverhalten zeige, dass die gemeinschaftsrechtliche Situation einer spezifischen Würdigung bedürfe. So führe auch die Betrachtung der Entwicklung im Bereich des europäischen Gesellschaftsrechts zu dem Schluss, dass der aktuelle Wettbewerb der mitgliedstaatlichen Gesetzgeber kein vertragsmäßiges Prinzip des EG-Vertrags darstelle. Vielmehr sei der gegenwärtige Zustand darauf zurückzuführen, dass die Gemeinschaftsorgane ihre Aufgabenstellung unterschiedlich interpretierten und die Rechtsangleichung im Bereich des Gesellschaftsrechts ins Stocken gekommen sei. Vor dem Hintergrund dieser Erkenntnis schilderte Teichmann abschließend die Folgen des Wettbewerbs der Rechtsordnungen sowohl auf Nachfrage- als auch auf Anbieterseite. So müsse vor allem die Anbieterseite, das heißt der nationale Gesetzgeber, flexibler werden und schnellere Entscheidungen als Reaktion auf neue Entwicklungen ermöglichen, was durch den Einsatz von Fachgremien erreicht werden könne. Schließlich führte Teichmann aus, dass das Informationsproblem eine besondere Herausforderung für die Gesetzgeber darstellt, dem aber durch die Schaffung eines einheitlichen Gesellschaftskollisionsrechts begegnet werden kann.

Die Darstellung der rechtlichen Grundlagen des europäischen Gesellschaftsrechts rundete Eva-Maria Kieninger mit ihrem Referat zur Notwendigkeit eines einheitlichen Gesellschaftskollisionsrechts ab. So bejahte Kieninger die Notwendigkeit einer solchen Regelung ausdrücklich. Die unterschiedlichen Reaktionen der Mitgliedstaaten auf die Rechtsprechung des Europäischen Gerichtshofes zur Niederlassungsfreiheit und zur Mobilität von Gesellschaften sei der erste Grund, warum Europa ein einheitliches Gesellschaftskollisionsrecht benötige. Zudem sei der Anwendungsbereich der Gründungstheorie immer noch nicht hinreichend geklärt, was sich vor allem darin äußere, dass im konkreten Fall die Anknüpfung an das Gesellschaftsstatut nicht klar von der Anknüpfung an das Delikts- oder das Insolvenzstatut abgrenzbar sei. Darüber hinaus wies Kieninger darauf hin, dass die Anknüpfung für Gesellschaften aus Drittstaaten ebenfalls der Klärung bedarf. Schließlich spreche auch die Tatsache, dass Wegzugskonstellationen, trotz der zu erwartenden Entscheidung des Europäischen Gerichtshofes in der Rechtssache Cartesio, immer noch nicht geklärt seien, für die Notwendigkeit eines Gesellschaftskollisionsrechts. Denn die Rechtssache Cartesio betreffe nur den Fall der tatsächlichen Sitzverlegung, während der Fall einer rechtlichen 
Sitzverlegung auch nach Cartesio ungeklärt bleibe. Auch den notwendigen Inhalt eines möglichen Rechtsakts stellte Kieninger unter Einbeziehung des Vorschlags des Deutschen Rates für Internationales Privatrecht für eine europäische Verordnung zum Gemeinschaftskollisionsrecht und des Referentenentwurfs zur Änderung des Einführungsgesetzes zum Bürgerlichen Gesetzbuch (EGBGB) vor. So müsse die Regelung hinsichtlich der Anwendung der Gründungstheorie - auch in Bezug auf Drittstaaten - uniform ausgestaltet werden. Bedenken gegen die Einbeziehung von Drittstaaten, etwa wegen mangelnder einheitlicher Schutzstandards der Rechnungslegung, könne durch eine entsprechende Norm, wie sie die aktuellen Entwürfe teilweise vorsehen, begegnet werden. Schließlich müssten auch das Gesellschaftsstatut und die Konstellationen der Sitzverlegung bei der Erarbeitung einer europäischen Regelung ausreichend gewürdigt und berücksichtigt werden. Kieninger wies abschließend erneut auf die Bedeutung eines einheitlichen Kollisionsrechts für die Mobilität von Gesellschaften hin. Dieses Kollisionsrecht könne auch als mögliche Basis für ein zukünftiges europäisches materielles Gesellschaftsrecht dienen.

Einzelfragen des europäischen Gesellschaftsrechts

Die Betrachtung einzelner Aspekte des europäischen Gesellschaftsrechts eröffnete Hanno Merkt mit seinem Referat zum Schutz der Gesellschaftsgläubiger im Binnenmarkt durch ein gesetzliches Mindestkapital. Zunächst stellte Merkt die Grundlagen des gegenwärtigen Systems der Kapitalaufbringung und Kapitalerhaltung dar. Dabei machte er deutlich, dass mittlerweile vor allem die bilanzielle Betrachtung im Rahmen der Kapitalerhaltung durch die in der zweiten gesellschaftsrechtlichen Richtlinie festgelegte Zielsetzung des Gläubigerschutzes einerseits und des Anlegerschutzes andererseits überfordert ist. Als Alternativen zum gegenwärtigen System erläuterte Merkt die Reformvorschläge der High Level Group, der Lutter-Gruppe sowie den Rickford Report und das Diskussionspapier des Instituts für Wirtschaftsprüfer in Deutschland e.V. Dem Konzept eines Mindestkapitals zum Schutz der Gläubiger werde danach nicht mehr die entscheidende Bedeutung beigemessen. Auch im Rahmen der Kapitalerhaltung werde eine mögliche Richtungsänderung weg von der bilanziellen Betrachtung hin zu einem Solvenztest vollzogen. Anschließend ging Merkt auf Grundund Einzelfragen einer möglichen Neuregelung des Gläubigerschutzes ein. Dabei stellte er unter anderem die Ausgestaltung des vertraglichen Gläubigerschutzes, wie er in den USA praktiziert wird, dem gesetzlichen Schutz der Gläubiger nach dem europäischen Modell gegenüber. Die Auswertung des Vergleiches beider Systeme führe dabei zu einem ,argumentativen Patt", sodass eine eindeutige Entscheidung für oder gegen ein bestimmtes System nicht mit Eindeutigkeit möglich sei. $\mathrm{Zu}$ unterschiedlich seien die beiden Schutzmechanismen in ihrer Zielsetzung und Ausgestaltung. Sicher sei nur, dass die kumulative Einführung eines vertraglichen und gesetzlichen Schutzsystems für die Gesellschaftsgläubiger überzogen und unflexibel wäre.

Rainer Krause gewährte einen Einblick in die Praxis des europäischen Gesellschaftsrechts, indem er sich den Fragen der grenzüberschreitenden Unternehmensmobilität widmete. Dazu stellte Krause zunächst die nationalen und europarechtlichen Grundlagen der Verschmelzung und Sitzverlegung von Gesellschaften dar. Die Verschmelzungsrichtlinie, die Rechtsakte zur Europäischen Aktiengesellschaft (SE), die Grundfreiheiten sowie die Rechtsprechung des Europäischen Gerichtshofes zur Niederlassungsfreiheit hätten die Mobilität von Gesellschaften durch Vereinfachung des Grenzübertritts erhöhen sollen. Die Praxis sehe jedoch vielfach anders aus. Krause ging zur Verdeutlichung auf einige „Mobilitätsbremsen" ein, die in der Realität die Verschmelzung und Sitzverlegung von Gesellschaften erschweren und schlug sogleich Lösungsmöglichkeiten vor. So stelle beispielsweise die Barabfindung für einen Aktionär im 
Falle des Widerspruchs gegen die Sitzverlegung die davon betroffenen Unternehmen vor Schwierigkeiten. Eine Barabfindung sei mit einem erheblichen Liquiditätsverlust verbunden und könne sich negativ auf die notwendige Aktionärsmehrheit auswirken, vor allem wenn die Barabfindung den aktuellen Börsenwert übersteigt. Diese Nachteile könnten eine Gesellschaft von einer vorteilhaften Sitzverlegung abhalten. Zudem fänden bei Barabfindungen die Vorschriften über den Erwerb eigener Aktien Anwendung, sodass die Gesellschaft darauf beschränkt sei, höchstens zehn Prozent des eigenen Kapitals von den widersprechenden Aktionären zu erwerben. Das Unternehmen sei aus diesem Grund darauf angewiesen, Vorkehrung zu treffen, um den Anteil der insgesamt widersprechenden Aktionäre unter zehn Prozent zu halten. Schließlich sorge auch die Tatsache, dass bei einem Übernahmeangebot auf das zu erstellende Prospekt und das Übernahmeangebot selbst unterschiedliches Recht anzuwenden ist, für einen nicht unerheblichen Nachteil. Krause machte mit seinen Ausführungen deutlich, dass die praktische Ausgestaltung der grenzüberschreitenden Mobilität noch einige Probleme bereitet.

Sodann ging Jochem Reichert auf die Erfahrungen der Praxis mit der SE ein. In einem ersten Schritt beschrieb er die tatsächliche Situation der SE, die mit insgesamt 130 Gründungen, davon 61 in Deutschland, mittlerweile breite Zustimmung gefunden hat. Dabei seien die Impulse zur Gründung einer SE nicht von den großen Unternehmen, sondern vom Mittelstand ausgegangen. Die Gründe für die Wahl der SE als Rechtsform wurden von Reichert ebenfalls erläutert. So spielten die gröBere Mobilität und eine erhebliche Flexibilität in der Organisationsverfassung eine entscheidende Rolle. Vor allem die Möglichkeiten, das monistische System zu wählen und den Aufsichtsrat zu verkleinern, sprächen eindeutig für die SE. Daneben sei aber auch das Image, wel- ches dem Unternehmen eine ,internationale Note" verleihe, für Gesellschaften attraktiv. In einem zweiten Schritt widmete sich Reichert einzelnen rechtlichen Problemen der SE. So beschäftigte er sich mit der Zulässigkeit von Vorratsgesellschaften, die er grundsätzlich bejahte. Vor allem das lange Gründungsverfahren einer SE spreche für die Zulässigkeit der Vorrats-SE. Daneben ging Reichert auch auf die Schwierigkeiten bei der Auslegung des Begriffs der strukturellen Veränderung im Rahmen des $§ 18$ Absatz 3 des Gesetzes über die Beteiligung der Arbeitnehmer in einer Europäischen Gesellschaft (SEBG) ein und befasste sich mit der Reichweite der Mitbestimmungsautonomie am Beispiel der Größe des Aufsichtsorgans. Abschließend stellte Reichert fest, dass die SE trotz einzelner ungeklärter Fragen in der Praxis eine gangbare Alternative darstellt. Der Freilandversuch lasse eine ,reiche Ernte erwarten“.

Mit dem Problem der Mitbestimmung bei grenzüberschreitenden Sachverhalten beschäftigte sich Martin Henssler. Den Ausgangspunkt seiner Ausführungen bildete dabei das deutsche Mitbestimmungsgesetz. Dieses erfasse keine ausländischen Rechtsformen, sodass zur Vermeidung der Mitbestimmung bis vor Kurzem auf eine ausländische Holding oder Kapitalgesellschaft und Co. KG habe ausgewichen werden müssen. Henssler erläuterte jedoch, dass die fortschreitende Europäisierung durch die Einführung der SE als Rechtsform und durch die Umsetzung der Mitbestimmungsrichtlinie im Gesetz über die Mitbestimmung der Arbeitnehmer bei einer grenzüberschreitenden Verschmelzung (MgVG) neue Möglichkeiten eröffnet habe, sodass heute kein deutsches Unternehmen gezwungen sei, ein System der paritätischen Mitbestimmung einzuführen. Im Anschluss daran stellte Henssler die Grundprinzipien der europäischen Mitbestimmung laut der Richtlinie 2005/56 $6^{3}$ dar und beschrieb ausführlich die

3 Richtlinie 2005/56/EG des Europäischen Parlaments und des Rates über die Verschmelzung von Kapitalgesellschaften aus verschiedenen Mitgliedstaaten, in: Amtsblatt der Europäischen Union, Nr. L 310 vom 25. November 2005, S. 1-9. 
möglichen Optionen der Geschäftsleitung eines Unternehmens im Falle, dass der Grundsatz, in der Konstellation des Hineinverschmelzens das Recht des Sitzstaates anzuwenden, nicht gelte. Die Richtlinie gewähre der sogenannten Verhandlungslösung den Vorrang, verhindere aber die Flucht aus der Mitbestimmung durch das „Vorher-Nachher-Prinzip“. Durch die Umsetzung der Richtlinie in das MgVG sei die Verhandlungslösung verdeckt in das deutsche Recht eingeführt worden, was vor allem Ausstrahlungswirkung auf die Reform des Mitbestimmungsgesetzes haben dürfte. Darüber hinaus ging Henssler auch auf einzelne Fragen der Wahl des besonderen Verhandlungsgremiums und des $\mathrm{Ab}$ schlusses der Mitbestimmungsvereinbarung ein. Im Anschluss stellte Henssler die Behandlung der Mitbestimmung in den Fällen des Hinausverschmelzens dar, um in einer abschlieBenden Bewertung strategische Vorschläge für ein Vorgehen bei grenzüberschreitenden Verschmelzungen anzubieten. Vorteilhaft erweise sich eine aus der Verschmelzung hervorgehende SE mit Sitz in Deutschland, soweit die SE zuvor als Holding mitbestimmungsfrei gegründet werden könne. Bei einer Verschmelzung ins Ausland sei dagegen die von der Richtlinie angebotene Lösung attraktiver.

Anstehende Projekte im Bereich des europäischen Gesellschaftsrechts

Thomas Bachner befasste sich anschließend mit der Aktionärsrechte-Richtlinie, ${ }^{4}$ wobei er zunächst Hintergrund und Zielsetzung erläuterte. Die Richtlinie habe die Erleichterung der grenzüberschreitenden Stimmrechtsausübung durch die Aktionäre im Blick. Dabei sei sie aber nicht auf die Möglichkeiten der Aktionäre zur Überwachung des Managements ausgerichtet, diese fänden sich bereits im nationalen Gesellschaftsrecht. Die Richtlinie sichere vielmehr ab, dass die Aktionäre diese Rechte auch effektiv umsetzen und im Rahmen der Hauptversammlung geltend ma- chen können. Die Richtlinie ziele dabei auf die Beseitigung bislang vorhandener Schwierigkeiten, welche sich etwa aus dem Hinterlegungserfordernis und dem damit verbundenen sogenannten share blocking, aus unzureichendem beziehungsweise verzögertem Informationszugang sowie dem komplizierten Verfahren zur Stimmrechtsausübung mittels Vertreter (sogenanntes proxy voting) ergeben können. In einem zweiten Schritt erörterte Bachner die materiellen Bestimmungen der Aktionärsrechte-Richtlinie. Besondere Aufmerksamkeit widmete er insoweit den Bestimmungen über die notwendigen Informationen vor der Hauptversammlung (Artikel 5), den Antragsrechten der Aktionäre (Artikel 6), der Umstellung vom Hinterlegungssystem auf den Nachweisstichtag (Artikel 7), der Möglichkeit zur Teilnahme an der Hauptversammlung auf elektronischem Weg (Artikel 8), dem Fragerecht (Artikel 9), der Stimmrechtsvertretung (Artikel 10-11), der Abstimmung per Brief (Artikel 12), der Erleichterung für Intermediäre (Artikel 13) und den Abstimmungsergebnissen (Artikel 14). Im dritten Teil seines Vortrages wandte Bachner sich ausgewählten Einzelaspekten der Aktionärsrechte-Richtlinie zu und erörterte unter anderem den Anwendungsbereich der Richtlinie, die Aktionärseigenschaft, die Gleichbehandlung der Aktionäre und den Nachweisstichtag. Zudem erläuterte er den ,,Fahrplan zur Hauptversammlung“, die Einberufung zur Hauptversammlung in ihrer Funktion als sogenannter information push und die Möglichkeit der elektronischen Teilnahme an der Hauptversammlung - etwa per Satellitenversammlung - und die damit verbundenen technischen und rechtlichen Risiken. Abschließend stellte Bachner ausführlich die nach Artikel 10 der Richtlinie gegebene Möglichkeit der Aktionäre vor, sich in der Hauptversammlung vertreten zu lassen. Es bestehe insoweit die Notwendigkeit der Aufhebung aller Beschränkungen in Bezug auf solche Personen,

4 Richtlinie 2007/36/EG des Europäischen Parlaments und des Rates über die Ausübung bestimmter Rechte von Aktionären in börsennotierten Gesellschaften, in: Amtsblatt der Europäischen Union, Nr. L 184 vom 14. Juli 2007, S. 17-24. 
die zu Vertretern bestellt werden können. Zudem dürfe die Anzahl der vertretenen Aktionäre nicht eingeschränkt werden, auch müsse das sogenannte split-voting zugelassen werden.

Paulina Dejmek erörterte sodann den Projektstand der Europäischen Privatgesellschaft. Dazu erinnerte Dejmek in einem kurzen historischen Rückblick an die Vorarbeiten zum derzeitigen Kommissionsentwurf der Verordnung über das Statut der Europäischen Privatgesellschaft (SPE-Verordnung). ${ }^{5}$ Dieser sei als Teil der Strategie der Europäischen Kommission für kleine und mittelgroße Unternehmen - die 99 Prozent aller Unternehmen in der Europäischen Union darstellten - aufgrund der Erkenntnis zustandegekommen, dass solche Unternehmen unter anderem wegen der unterschiedlichen Rechtssysteme, der abweichenden Steuer- und Mitbestimmungsregelungen und der Skepsis gegenüber unbekannten Gesellschaftsformen bei ihrer Expansion in den Binnenmarkt gehindert seien. Dejmek erläuterte anschließend zunächst die Hauptzüge der Verordnung. Als Rechtsgrundlage komme allein Artikel 308 EGV in Betracht, da wegen der Rechtsprechung des Europäischen Gerichtshofes ${ }^{6}$ bei Schaffung neuer Rechtsformen insbesondere eine Anwendung von Artikel 95 EGV ausscheide. Die Europäische Privatgesellschaft sei als juristische Person mit Rechtspersönlichkeit ausgestaltet, deren Anteilseigner begrenzt hafteten und welcher der Börsengang verwehrt sei. Als Konsequenz der Rechtsprechung des Europäischen Gerichtshofes zur Niederlassungsfreiheit müssten jedoch die Hauptverwaltung und der eingetragene Sitz der Europäischen Privatgesellschaft nicht im selben Mitgliedstaat ansässig sein. Im Vergleich zur SE verfüge die Europäische Privatgesellschaft über ein selbstständiges Statut. Anders als bei der SE sei auch eine Neugründung möglich, auch auf die Anforderung des grenzüberschreitenden Elementes bei Grün- dung der Europäischen Privatgesellschaft würde verzichtet. Zudem sei nach dem derzeitigen Entwurf der Verordnung kein Mindestkapital vorgesehen. Sodann gab Dejmek einen Überblick über die Vorschriften der Verordnung und deren Anhang, welcher eine Liste von Punkten enthält, welche die Gesellschafter zwingend in der Satzung regeln müssen. Die Verordnung enthalte unter anderem Vorschriften über die Gründung (Artikel $5 \mathrm{ff}$.), die Gesellschaftsanteile (Artikel 14 ff.), das Gesellschaftskapital (Artikel 19 ff.), die interne Organisation (Artikel 26 ff.), die Mitbestimmung (Artikel 34), die Sitzverlegung (Artikel 35 ff.) und die dann geltenden Mitbestimmungsregeln (Artikel 38). Insgesamt sehe die Verordnung ein hohes Maß an Flexibilität - insbesondere hinsichtlich der Gestaltung der internen Organisation - vor, welche gewissen Einschränkungen - etwa im Bereich des Minderheitenschutzes - unterliege. Abschließend ging Dejmek auf mögliche Probleme im Hinblick auf den Erlass der Verordnung ein. Schwierigkeiten sah sie dabei unter anderem im Hinblick auf die Anwendung des Artikels 308 EGV und das Fehlen eines grenzübergreifenden Anknüpfungspunktes.

Den neuesten Entwicklungen im Bereich des europäischen Verbands- und Stiftungsrechts widmete sich zum Abschluss der Tagung $S \phi$ ren Friis Hansen. Nach einem kurzen Abriss der geschichtlichen Entwicklung der Europäischen Stiftung, ging Hansen sodann ausführlich auf den Inhalt der von der Arbeitsgruppe zum European-Foundation-Project vorgeschlagenen Verordnung zur Schaffung einer Europäischen Stiftung ein. Diese sei zweigeteilt und beinhalte sowohl zivilrechtliche als auch steuerrechtliche Aspekte. Im zivilrechtlichen Verordnungsteil befinde sich in Artikel 9 ein Bekenntnis zur Gründungslehre, in Artikel 1.1 eine Definition des Stiftungsbegriffes, in den Artikeln 4 und 5 Regelungen zur Stiftungsorganisation und in Artikel 1.2 Normen den Stiftungszweck betreffend. Zudem sei im

5 Europäische Kommission: Vorschlag für eine Verordnung des Rates über das Statut der Europäischen Privatgesellschaft, $\operatorname{KOM(2008)~} 396$.

6 Vgl. Europäischer Gerichtshof: Rs. C-436/03 (Europäisches Parlament/Rat der Europäischen Union), 2006. 
Verordnungsvorschlag die Frage der Unternehmensstiftung und der staatlichen Stiftungsaufsicht berücksichtigt. Der steuerrechtliche Teil des Verordnungsvorschlages sehe ein Model-Tax-Law und stiftungszweckorientierte Steuerbefreiungen vor, obwohl mit dem Stauffer-Urteil ${ }^{7}$ eigentlich der Weg für alternative sogenannte Non-Discrimination-Rules geebnet sei. Vom Verordnungsvorschlag seien derzeit weder Mitbestimmungsfragen noch Regelungen zur Sitzverlegung oder zu grenzüberschreitenden Aktivitäten erfasst. Ob die Europäische Stiftung tatsächlich eine Zukunft habe, sei letztlich eine politische Frage. Hansen betonte erneut die Bedeutung des gemeinnützigen Sektors in der Europäischen Union und wies auf die tatsächlichen Behinderungen für die grenzüberschreitende Aktivität von Stiftungen hin. Letztlich hinge die Zukunft der
Europäischen Stiftung aber maßgeblich vom Erfolg der derzeit in der Entstehung begriffenen Europäischen Privatgesellschaft ab.

\section{Ausblick}

Die Vorträge und Diskussionen im Rahmen der Tagung zeigten eindrucksvoll die Dynamik des europäischen Gesellschaftsrechts auf. Vor allem die Notwendigkeit eines einheitlichen Gesellschaftskollisionsrechts ist in der derzeitigen Diskussion als besonders dringend zu bewerten. Die erwünschte grenzüberschreitende Mobilität für Gesellschaften kann nur erreicht werden, wenn die tatsächlichen und rechtlichen Hemmnisse abgebaut und die Harmonisierung der rechtlichen Standards mit dem Ziel des einheitlichen Binnenmarkts weiter vorangetrieben werden.

7 Europäischer Gerichtshof: Rs. C-386/04, (Centro di Musicologia Walter Stauffer/Finanzamt München), Vorabentscheidungsersuchen des deutschen Bundesfinanzhofes, 2006. 